\title{
AN APPROACH TO SENTENCE-LEVEL ANAPHORA IN MACHINE TRANSLATION
}

\author{
Gertjan van Noord, Joke Dorrepaal, Doug Arnold \\ Steven Krauwer, Louisa Sadler, Louis des Tombe \\ Foundation of Language Technology \\ State University of Utrecht \\ Trans 103512 JK Utrecht \\ Dept of Language and Linguistics \\ University of Essex, Wivenhoe Park, \\ Colchester, C04 3SQ, UK. \\ February 15, 1989
}

\begin{abstract}
Theoretical research in the area of machine translation usually involves the search for and creation of an appropriate formalism. An important issue in this respect is the way in which the compositionality of translation is to be defined. In this paper, we will introduce the anaphoric component of the Mimo formalism. It makes the definition and translation of anaphoric relations possible, relations which are usually problematic for systems that adhere to strict compositionality. In Mimo, the translation of anaphoric relations is compositional. The anaphoric component is used to define linguistic phenomena such as wh-movement, the passive and the binding of reflexives and pronouns mono lingually. The actual working of the component will be shown in this paper by means of a detailed discussion of wh-movement.
\end{abstract}

\section{Introduction}

Theoretical research as part of machine translation often aims at finding an appropriate formalism. One of the main issues involved is whether the formalism does full justice to the idea that the translation of a whole is built from the translation of its parts on the one hand and whether it leaves enough room for the treatment of exceptions on the other hand. In other words, the question is in what way the idea of compositionality is to be defined within a particular formalism. An answer to this question from an interlingual perspective is given in the literature on the
Rosetta system (e.g. Landsbergen 1985). The CAT frame work (e.g. Arnold et al. 1986) was meant to be an an swer to the same question, this time for a transfer system viz. the Eurotra system. The MiMo formalism is a re action to the CAT framework and tries to solve severa translation problems by formulating an alternative defini tion of compositionality. Phenomena involving anaphor 1 such as wh-movement and the coindexation of pronomi nals often cause problems for strictly compositional system since translation of one word depends on (the translation of) another word, one which can be quite far away in th sentence. Rosetta tackles this problem by distinguishin between rules that are significant with respect to the com positionality of translation, so-called meaningful rules, an rules that are not, referred to as transformations (Appel et al. 1987); in this way the system is not compositional is the strict sense anymore. The notion of compositionalit: MiMo adheres to is defined in such a way that anaphori relations can be translated compositionally as well. In thi paper we will introduce the anaphoric component of th MiMo formalism. It is used to define linguistic phenomen such as wh-movement, the binding of reflexives and pro nouns, the passive and control phenomena monolingually The formalism will be discussed by means of an extensiv description of a possible analysis of wh-movement.

In the next section, we will first discuss and motivate som of the more fundamental characteristics of the MiMo trans lation system. Section two will sketch the MiMo formalisn

\footnotetext{
${ }^{1}$ In this paper the term 'anaphoric' should be interpreted in th broadest sense, as opposed to Chomsky 1981 in which only A-trace and reflexives are called anaphoric.
} 
as far as necessary for understanding what will follow. The component that deals with the treatment of anaphora will be discussed in section 3 . In the fourth section the actual working of the component will be shown by an elaborate discussion of wh-movement. Finally, the translation of anaphoric relations will be defined and some idea will be given of the kind of problems that remain and that will have to be subject to further research.

\section{MiMo}

The MiMo formalism tries to come up with an answer to the question what compositional translation should imply. Strictly compositional systems have to deal with several translation problems. As to what these problems exactly are depends on the nature of the definition of the notion compositionality. In general, two kinds of problems can be distinguished. First, there are the problems that arise when languages do not really match. Second, the problems that occur when translations of two constructions depend on one another.

The former type of problem is caused by lexical and structural holes. It means that source and target representation do not really match. Lexical holes occur when a language lacks words equivalent to the ones in the source language. In the case of structural holes, the target language lacks an equivalent construction rather than a word. A description of the concept will have to be used in these cases. For an example of a lexical hole, compare sentence (1) and its translation into English (2).

(1) Jan zwemt graag

(2) John likes to swim

Unlike sentences with an adverb like 'vandaag', (1) cannot be translated compositionally in the strictest sense. The translation of (1) is not simply the translation of the parts the constituent is composed of. This problem has been solved in the CAT framework by liberalizing the definition of compositionality in such a way that it will be possible to render (1) directly into (2), by means of a rule like (3).

(3) $r 1(s 1, s 2$, graag $) \Rightarrow r 2(t(s 1), r 3($ like,$t(s 2)))$

By (3) a construction composed of three daughters, s1, s2 and 'graag' will be translated into a construction having two daughters, viz. the translation of $s 1$ and a construction that again has two daughters, that is, the verb 'like' and the translation of 82 . The main disadvantage of this approach is the fact that combinations of exceptions have to be described explicitly again, see (4) and (5).
(4) Jan zwom gewoonlijk

John used to srim

(5) Jan zwom gewoonlijk graag

John used to like to swim

The translation of 'gewoonlijk' requires a rule simi (3). However, a combination of 'graag' and 'gewo appears to be possible as well. An additional rule wil to account for this. This will lead to an enormous exp of the number of rules. It is one of the main reasons alternative definition of compositionality within the system. The nature of the definition allows the trans of both 'gewoonlijk' and 'graag' in case they coocc translation rule separates a constituent into an orc part and an exceptional part. Both parts are then lated separately and finally, in the target language, th translated parts are joined again. In the case of a ser consisting of both 'graag' and 'gewoonlijk', the ser is separated into an exceptional part, 'graag' for exa and an ordinary part, the rest of the sentence. Thi again is separated into an exceptional, 'gewoonlijk', a ordinary part. The latter is again that which is le hind after extraction of the exceptional part. In th all these parts are joined and will make up a constr in the target language. So, in MiMo not all daughte translated in one shot but part of a constituent is tran while the rules can still work on the rest of the consti An extensive discussion of problems like these is to be in Arnold e.a (1988).

The second type of problems w.r.t compositional translation involves translation of phrases that are ally dependent. Examples hereof are translations of $\mathrm{pl}$ that are anaphorically linked. Translation requires these relations are established. Examples are to be in (6). In (6), the relation between the subject and $t$ flexive pronominal is necessary to arrive at the correc 1 of the reflexive pronominal in French. In (7), knowle the functional status of the wh-word is relevant to $b$ to generate the right case in German.

(6) the women think of themselves $\Rightarrow$

les femmes pensent a elles-memes/*ils-memes

(7) who $\operatorname{did}$ you see $\Rightarrow$ wen $/{ }^{*}$ wer $/{ }^{*}$ wem sahest du

In this paper we will examine the component of the formalism that has been developed to enable the for tion of anaphoric relations on the one hand and con tional translation on the other. The system disting itself from other systers $s$ in the field of computation. guistics, such as GPSG (Gazdar et al. 1985), PAT] e.g. Shieber 1986) and DCG (Pereira and Warren for its central notion of modularity. The formalism ex 
'anaphor'. I-structures having this feature will have to be bound by an antecedent in the end. Examples of these are pronouns and reflexives. This requirement also holds for empty slots. They are considered anaphoric and will have to be bound as well unless we deal with optional slots. Binding of I-structures happens through anaphoric rules. In the next section we will show the way these rules are formulated. The final structure of (13a) will be (14). In (14), a relation between the topic (I1) and the embedded subject position (I2) ${ }^{4}$ is established ${ }^{5}$. The subordinate

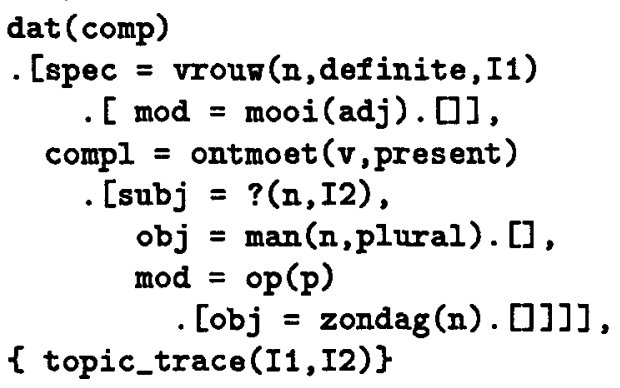

complementizer is also regarded as a lexical word. Even sentences that do not show a complementizer at surface are assigned one. This is not in any way intrinsic to MiMo but makes a uniform account of several phenomena possible. This type of complementizer has two slots: an optional slot for topics or wh-words and a slot for a verb construction.

\section{The definition of anaphoric rela- tions}

Anaphoric relations are defined by a type of rule that is quite different from the ordinary rules. This distinguishes the system from, for example DCG. With PATR and DCG the possibility of percolation from, say topic to trace, influences all the other rules. MiMo's approach, a separate type of rule for the anaphoric component, has the advantage of leaving the other rules, i.e the lexical I-structures, as they are. Modularity is one of MiMo's qualities. This quality is also considered important in GPSG (Gazdar et al. 1985) where it is realized by the use of metarules that multiply the number of rules. This would be undesirable in MiMo

\footnotetext{
41 and I2 are unique names which are automatically assigned to every I-structure. We will indicate them henceforth as capitalized words. Names to which no further reference is made will be omitted for clarity's sake. An I-structure consists of a tree and a set of anno tations that denote the anaphoric relations within the tree. The tree annotated with this set will be called I-object henceforth.

${ }^{8}$ Note that we will usually leave out optional slots that are not filled
}

since every lexical word is its own rule. So then eve number of words would have to be multiplied.

The use of a different rule type is also motivated $b$ process of translating anaphoric relations. If we only feature percolation to encode anaphoric relations, the tions established would not be explicit anymore. Ar tions in MiMo are clearly distinguishable from the $\mathrm{I}$ the representation and as such make it possible to de compositional translation of them in transfer.

Besides being modular, the system also proves to be $d$ ative. Both qualities, modularity and declarativit: hance the workability for the user. Changes and sions are quite easily achieved and rules can be defir a general way. An anaphoric component written fc particular language can often be used for another lan with minor changes.

Anaphoric rules create anaphoric relations with structures. This has two consequences in our system. first place, some of the features of antecedent and an are unified. These features are called 'transparent'. for example, makes it possible to define agreement ph ena. The linguist defines which features are transr with respect to a certain rule. The motivation for th proach is discussed at length in Krauwer et al. (1987) main point is that identity of some but not all featr required in an antecedent-anaphor relation. In the $s$ place, the I-structure is augmented with an annotation specifies the binding. This annotation consists of the of the relation and the unique names of the nodes be which the relation exists. The definition of anaphos lations makes use of these annotations (see also secti A relation cannot be created unless the correct stru relation between antecedent and anaphor exists. $\mathbf{S}$ grammar writer defines for each relation:

1) the name of the relation

2) the transparent features

3) the structural relation

An example of an anaphoric rule is the one that 1 lishes a relation between a wh-element and an oper The rule looks like (15) ${ }^{6} \mathrm{MiMo}^{7}$.

$$
\begin{aligned}
\text { wh_trace : } & c_{-c o m m a n d}\{w h\},\{\text { open\} })- \\
& \text { \{agreement, case }\}
\end{aligned}
$$

The wh-trace relation is established when the stru relation c-command holds between a wh-constituen

\footnotetext{
${ }^{6}$ In fact, the wh-trace relation is subject to more restriction c-commandment. We will return to this in section 4.

'A special feature 'open' is used to refer to open slots. A have this feature by default as long as they are not filled. So, can be regarded as a feature of the trace since slots not (yet can be considered potential traces.
} 
an open slot. The agreement features and the case feature are unified if possible, if not, the relation will not be established. The structural relation itself, c_command in this case, is defined by the user as well. Either a simple structural relation is defined or a complex structural relation. The latter is composed of a regular expression over structural relations ${ }^{8}$. An example of a simple structural relation is the sister-relation, defined in (16).

$$
\begin{aligned}
& \text { (16) sister (ANT, ANA) : } \\
& \text { ?() } .[?=\text { ? (ANT), } \\
& ?=?(\text { ANA })]
\end{aligned}
$$

The structural relation sister holds between the I-structures ANT and ANA if there exists an I-structure in which both ANT and ANA fill slots. The exact nature of the LIs is not important nor are the features or the names of the slots, hence their representation as question marks in $(16)^{9}$ - A complex structural relation is defined by means of a regular expression over structural relations. The regular expressions make use of the operators "', indicating optionality, ';' for disjunction, '*' for iterativity (0, 1 or more times ) and ' + '. The latter has a special meaning which can best be explained by means of the definition of the c_command relation mentioned in (17). The ' + ' operator indicates that the sister relation should hold between the antecedent and some intermediate node and the ancestorrelation between this intermediate node and the anaphor. The Prolog-variant of (17) is (18). So, the c_command relation holds between the I-structures ANT and ANA when one of ANT's sisters is ANA's ancestor. The MiMo defini-

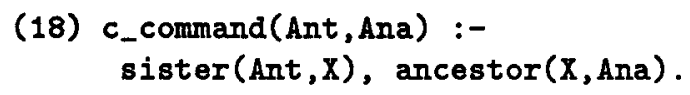

tion of 'ancestor' is given in (19a). The relation is defined in terms of the simple relation 'mother'. The structural relation of the latter is in $(19 b)^{10}$. Features can be added to the structural pattern to restrict the range of possible relations further. This will be illustrated in the fourth section when we discuss a possible way of treating wh-movement. To

\footnotetext{
'This idea is partly based on LFG's notion of functional uncertainty. See Kaplan et al. 1987.

Note that the order of ANT w.r.t ANA is not relevant since the order of the slots is not in any way related to word order in the sentence.

${ }^{10}$ All I-structures are also their own ancestor according to the definition in (19a). This is the correct result when used in the c_command definition since sisters do c_command one another. In case this is undesirable however, the relation could be defined as follows :

ancestor : mother $+{ }^{*}$ mother

Generally, the correct definition of a relation like c_command depends of course on the use it's being made of in anaphoric rules and on the make up of the I-structures used. The definition above should merely be regarded as an exemplification of the mechanism.
}

(19) a. ancestor : * mother

b. mother (ANT, ANA) :

?(ANT).[? = ?(ANA)]

conclude this section, we give an example of an I-structur to which (15) applies. (20b) shows the structure before an (20c) after application of (15).

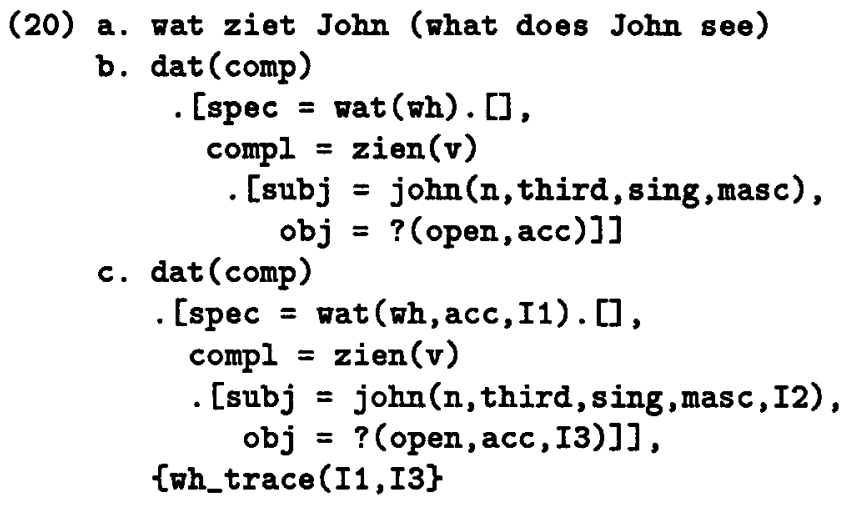

\section{WH-Movement}

In this section, the actual working of the anaphoric compc nent will be discussed. We will do this by showing how linguistic phenomenon like wh-movement could be imple mented. Note that none of the linguistics in this sectio: follows from the system. The aim of the discussion is $t$ give an idea of the power of the anaphoric component and $c$ the kinds of linguistics that can be put to use. We will firs introduce the linguistic environment and present some dat. from Spanish that reflect some of the surface phenomen caused by the presence of anaphoric relations. The sectios on the implementation of the wh-relation will argue tha and show how surface phenomena of this nature can $b$ handled deterministically.

\subsection{Introduction}

The wh-trace relation seems the most interesting one be cause it shows both how general and powerful the mecha nism is and how restrictive the rules should be to accoun for the data. At least the data shown in (21) should b accounted for. In the GB framework (e.g. Chomsky 1981) wh-movement is seen as an instance of the transformatio 'move alpha', which respects the subjacency principle. Th 
(21) a. why do you think John left (ambiguous)

b. who do you think Bill told me ... Susan

c. *who do you believe the claim that Bill sar - (violation complex NP constraint)

d. *who do you know whether - left (violation wh-island constraint)

e. *who did you whisper - came (non-bridge verb) said - was ill (unbounded dependency)

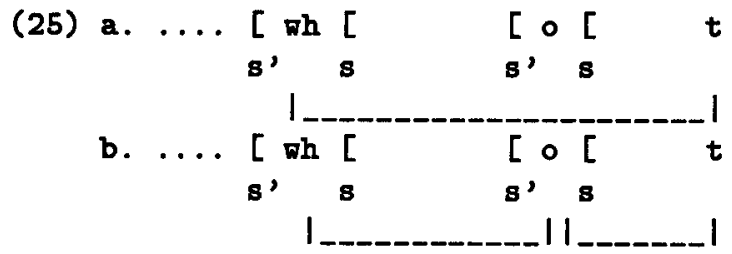

of complementizers.

\subsection{Implementation}

The structural relation for wh-movement should refl idea that the wh-constituent may bind across one k ing node at most. Note that, before and after the cr of this bounding node, it may theoretically cross an ited number of nodes that are not bounding. The tural relation that reflects this idea looks like (26k wh-trace relation is defined in (26a). The wh-trac

For English, S and NP are assumed to be bounding nodes. Wh-movement takes place cyclically via the comp-positions of the intermediate clauses, leaving behind traces (the socalled comp-to-comp movement). As such, it does not cross more than one bounding node at a time in a structure like (23).

Our discussion of wh-movement in the next section is in accordance with the comp-to-comp movement. Although other approaches, such as direct movement, are feasible too, we will adhere to the comp-to-comp approach. Data from Spanish (Torrego 1984) also seem to support the preference for actual movement from complementizer to complementizer.

(24) Que [ dice Juan [ que [ creian los dos [ que [ habia pensado Pedro [ que [ habia aplazado el grupo [ el grupo habia aplazado

What says John that thought the two that believed Peter had postponed the group; that the group had postponed

According to Torrego, inversion is obligatory in all clauses except the lowest. In the lowest clause, inversion is optional. The GB theory accounts for this by claiming that for Spanish S-bar, instead of $S$, is the bounding node. This predicts that movement in the lowest cycle can take place in two ways, as shown in (25). Neither of the two violates subjacency. Assuming that a wh-constituent, or its trace, in comp triggers inversion, the variation in Spanish wordorder in the lowest cycle is accounted for.

We will return to these data in the next section. We will argue that these data can be handled by the MiMomechanism as well, given the correct rules for the binding

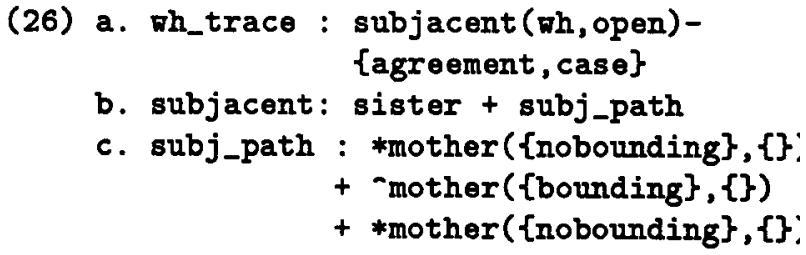

tion is established by the structural relation subjace tween a wh-element and an open slot. The definition subjacent-relation closely resembles that of c_com Instead of the relation 'ancestor', a relation 'subj_p defined that specifies a path consisting of one bot node at most. Non-bounding nodes may invervene Subjacency then is not defined as a filter, it is a $p$ formulation of possible relations. Note that (26) is both for languages in which $\mathbf{S}$ is a bounding node, $\mathbf{s}$ English, and for languages which have S-bar as bor node. The difference in boundedness will be expres the lexicon and the bindings will be established acc to the definition of subjacency and given the bound, of particular nodes ${ }^{11}$.

As has been shown in (25a) and (25b), the trace can $\varepsilon$ be bound in two ways in languages that have S-ba bounding node, provided there are at least two clau between the antecedent and the trace. We can make

\footnotetext{
${ }^{11}$ The difference between bridge verbs and other verbs is a coded in the lexicon. Only bridge verbs allow comp-to-comp ment. The generalization might be expressed by asaigning the bounding to sbar complements and modifiers in all other case this, sbar is a bounding node in some cases too.
} 
use of this in MiMo. The Spanish synthesis component can check whether the comp-position of a clause is either filled or bound. If so, the clause is inverted. In this way, the variation in word order in Spanish wh-questions will be quite naturally accounted for.

This leaves us to show that our definition of wh-trace indeed establishes a relation in two different ways between the antecedent and the open position. (27b) shows the MiMo version of the structure in (27a). (27c) indicates the way in which the relation is found without binding the complementizer in the embedded clause. The relation 'sister' holds between the antecedent and the node 'pensado'. Ths node in its turn binds the open position I3, through mother-relations. The movement involves the crossing of one bounding node. (27d) indicates the relation found.

\section{(27)}

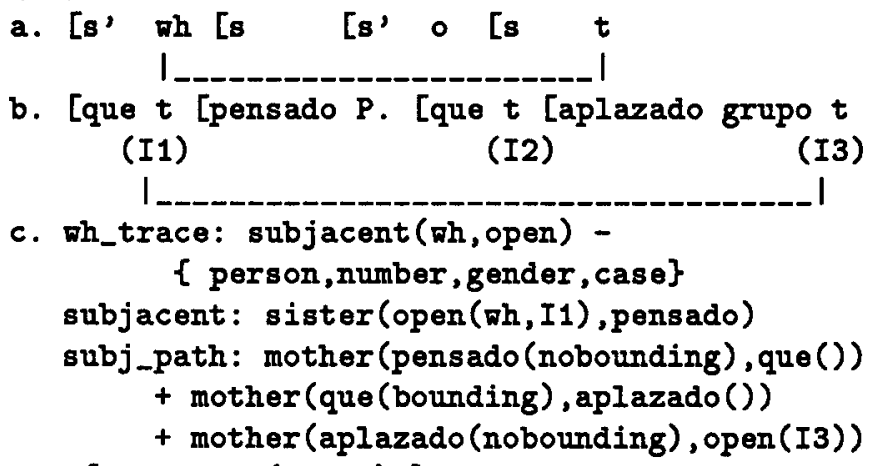

(28) shows that two relations can be found. The GB structure and the MiMo structure are shown in (28a) and (28b) respectively. In (28c1), the relation between I1 and I2 is found and (28c2) shows the one between I2 and I3. Both relations are mentioned in $(28 \mathrm{~d})$.

In (28), the intermediate empty complementizer-position is bound, hence inversion will take place. In (27) the complementizer is neither filled nor bound, so no inversion in this case. The data are accounted for in quite a natural and linguistically sound way. They are the direct consequence of the definitions of structural relations and they do not have to be generated by some kind of arbitrary inversion mechanism.

\section{The translation of anaphoric rela- tions}

In this section, we intend to give an impression of the usefulness of coindex relations in translation and the translation of the relations themselves. In linguistics, a monolin-
(28)

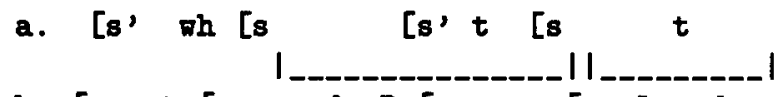

b. [que $t$ [ pensado $P$ [ que $t$ [ aplazado grupo $t$ (I1) (I2) (I3

c.1. wh_trace: subjacent (wh,open)\{person, number, gender, case\}

subjacent: sister (open ( $\mathrm{hh}, \mathrm{I} 1)$, pensado)

subj_path: mother (pensado(nobounding), que()

+ mother (que(bounding), open(I2))

2. vh_trace: subjacent (vh,open)\{person, number, gender, case\}

subjacent: sister (open (wh, I2), aplazado)

subj_path: mother (aplazado(nobounding), open (I3))

d. $\left\{\nabla h \_t r a c e(I 1, I 2), \nabla h \_t r a c e(I 2, I 3)\right\}$

gual account of coindexation is quite an achievement. In machine translation, the most important part of research deals with the translation of the relations that were estab. lished monolingually.

The I-object to be translated consists of an I-structure an. notated with anaphoric relations. An I-object is the result of the application of certain anaphoric relations (denoted by the annotations) to a particular I-structure. The com. positional translation of an I-object is the result of the application of the translated annotations to the translated I-structure. We hold the view that anaphoric relations are universal in MiMo. The translation of a relation between the I-structures $I$ and $J$ is that same relation between the translations of $I$ and $J$. This is summarized in (29).

(29) the translation of an I-object:

The translation of an I-object I1 is the result of the application of the translations of the annotations of I1 to the translation of I1's I-structure. The translation of an annotation $R(I, J)$ is $R(t(I), t(J))$.

The final set of anaphoric relations of the target object should be equivalent to the set that existed at the source level. The following example illustrates principle (30) :

(30) Por que [ dice Juan [ que [ los dos creian [ que [ Pedrc habia pensado [ que [ el grupo habia aplazado la reunion Why say John that the two thought that Peter believed that the group postponed the meeting

Inversion being obligatory in all clauses except the lowest, 'por que' can only bind the modifier position in either the first or the second clause. Each relation further down is ex- 
cluded as more clauses would have to show inversion then. When we ignore the bindings established at the Spanish I-level, translation into English will produce a lot of possible translations since 'that' may or may not be inserted in every complementizer position in English. However, the impact of this complementizer on possible anaphoric relations is not totally irrelevant. According to WAHL (1987), the complementizer blocks binding of 'why' to an empty position deeper down, cf. (31) and (32).

(31) why(i)/(j) do you think _(i) the boat sank _(j)

(32) why(i) do you think -(i) that the boat sank -

When we preserve the bindings from Spanish and we claim that in English 'that' may never be inserted when its modifier position is bound to an antecedent, we can deterministically arrive at the right translation :

(33) Por que [ dice Juan [ que [ los dos creian [ que [ Pedro habia pensado [ que [ el grupo habia aplazado la reunion

(34) Why [ did John say [ [ the two thought [ that [ Peter believed [ (that) the group had postponed the meeting

Both are ambiguous since both can question the reason for John's 'saying it' and 'the two believing it'. Other interpretations are excluded in both Spanish and English.

Definition (29) also causes some problems. Take the following example from Italian (cf. Chomsky 1981) :

(35) l'uomo [che mi domando [chi abbia visto]] the man(i) of whom I wonder who(j) e(i) saw $e(j)$

One might wonder what the English translation would have to be in the first place. In MiMo, the incorrect literal translation will not be found because the necessary anaphoric relations cannot be established. In cases like these, separate translation rules are needed to arrive at a translation of (35). It is possible to refer explicitly to anaphoric relations as long as they are restricted in depth. This is necessary in case an expression without anaphoric relations translates into one which requires a linking between an antecedent and an anaphor. An example is (36).

(36) Jan zwemt graag $\Rightarrow$ John(i) likes _(i) to swim

Unboundedly deep embedded relations are however not accessible by translation rules in the transfer component. Another problem we face deals with the interaction of anaphora and other standard 'non-compositional' phenomena, such as the example of Dutch 'graag' translating as 'to like' in English (see section 1). These examples, as well as anaphora, can be handled compositionally, as we have shown. The interaction however poses some problems, see
(37).

(37) Hoe graag zwom Jan $\Rightarrow$ How much did John swim

Since 'graag' is displaced, translation of 'graag' as t ceptional part of the embedded sentence is not pc given that the movement is not undone $e^{12}$. These are even noncompositional from MiMo's tolerant $v$ compositionality.

\section{Conclusion}

In this paper we showed the need for a non-standard of compositionality in translation. With the MiMo tion of compositionality we are able to define the ti tion of sentence level anaphora. In MiMo, anaphori tions are defined by a separate type of rule. This e linguists to define anaphoric relations in a declarati modular way. It appeared that linguistic generaliz can be defined quite naturally and generally. It is the linguist to decide which generalizations are to $t$ ferred and how they can best be expressed. We ch formulate principles in a general way. The relation' cent' was meant to serve all languages. Restrictions, * semantic features, can be added freely. The definitic late to information that is encoded in the language-s lexicon. This produces the variations that exist acro: guages.

The use of a separate type of rule enables a compos: definition of the translation of anaphoric relations $b_{1}$ the applied rules are still visible - as annotations structure to be translated. The translation of an Iwas defined as the translation of the I-structure to the translations of the anaphoric rules applied. The lation of an anaphoric rule is the target equivalent $c$ rule. This point of view poses problems in cases whe source language is less restrictive than the target lan! In that case, special rules have to be written to as translation nonetheless. When a particular relation also : interpreation) has been established in the sourc guage, it should be present in the target language interpretations should be translated of course. This yet possible in the current system when unboundedly relations need to be seen in the transfer component.

\footnotetext{
${ }^{12}$ It is of course also possible to assume that all wh-movemen been undone. In Mimo, this only means a shift of problems $f$ transfer to the analysis and synthesis modules. Besides, th would still hold for other long-distance phenomena like prono
} 


\section{Acknowledgements}

The work we report here had its beginnings in work within the Eurotra framework. MiMo however is not "the" official Eurotra system. It differs in many critical respects from e.g Bech \& Nygaard (1988). MiMo is the result of the joint effort of Essex, Utrecht and Dominique Petitpierre from ISSCO, Geneve. The research reported in this paper was supported by the European Community, the DTI (Department of Trade and Industry) and the NBBI (Nederlands Bureau voor Bibliotheekwezen en Informatieverzorging).

\section{References}

L Appelo; C Fellinger; J Landsbergen, 1987: "Subgrammars, Rule Classes and Control in the Rosetta Translation System" in: European Chapter ACL. 1987 Copenhagen.

D Arnold; S Krauwer; M Rosner; L des Tombe; G Varile, 1986: "The CAT framework in Eurotra: A theoretically committed notation for MT $^{n}$. in: Proceedings of Coling. Bonn 1986.

D Arnold; S Krauwer; L des Tombe; L Sadler, 1988: "Relaxed compositionality in Machine Translation". in: Second International Conference on Theoretical and Methodological issues in Machine Translation of Natural Languages Carnegie Mellon Univ. Pittsburgh 1988.

A Bech; A Nygaard, 1988: The E-framework: a formalism for natural language processing". in: Proceedings of Coling. Boedapest 1988.

J Bresnan (ed) 1982: The Mental Representation of Grammatical Relations. Cambridge MIT press. 1982.

N Chomsky 1981: Lectures on Government and Binding. Foris Dordrecht, 1981.

G Gazdar; E Klein; G Pullum; I Sag, 1985: Generalized Phrase Structure Grammar. Blackwell Publishing and Cambridge Mass. 1985.

R Kaplan; J Maxwell; A Zaenen, 1987: "Functional Uncertainty". CSLI Monthly vol 2, no 4 january 1987.

S Krauwer; M King (eds), 1987: The Eurotra Reference Manual 3.0

J Landsbergen, 1985: "Isomorphic Grammars and their use in the Rosetta Translation system". in King, M (ed) Machine Translation Today Edinburgh university press 1985.

F Pereira ; D Warren, 1980 : "Definite Clause Grammars for Language Analysis - A Survey of the Formalism and a Comparison with Augmented Transition Networks". Artificial Intelligence 13 F Pereira ; S Shieber, 1987: Prolog and Natural Language Analysis. CSLI 1987.
S Shieber, 1986: An introduction to unification based approaches to grammar. CSLI 1986.

E Torrego, 1984: "On Inversion in Spanish and Some of Its Effects", Linguistic Inquiry 15, 103-130.

WAHL, 1987: J Aoun; N Hornstein; D Lightfoot; A Weinberg: "Two types of locality" Linguistic Inquiry 18, 4. 